\title{
Caracterização epidemiológica do câncer do colo uterino, anterior à implantação do calendário vacinal para o HPV no estado do Maranhão
}

\author{
Epidemiological characterization of cereal cancer, previous to the implementation of the \\ vacinal calendar for HPV in the state of Maranhão
}
Caracterización epidemiológica del cáncer de cervix uterino anterior a la implementación del calendario vacinal para VPH en el estado de Maranhão

Luana Mendes Nogueira ${ }^{1}$, Sueli de Souza Costa ${ }^{1}$, Izolda Souza Costa ${ }^{2 *}$, Deyvison de Lacerda Lopes $^{1}$, Daizy Carlota Coelho Aguiar ${ }^{1}$, Luís Carlos Machado e Silva1.

\section{RESUMO}

Objetivo: Identificar e analisar o perfil epidemiológico do câncer do colo do útero anterior à implantação do calendário vacinal para o HPV no Estado do Maranhão, no período de 2006 a 2014. Métodos: Foram analisados dados epidemiológicos encontrados no SISCOLO/DATASUS, e IBGE, de oito macrorregiões de saúde, com as variáveis: índices sociodemográficos (escolaridade, raça/cor, IDH); indicadores em saúde (número de exames coletados, número de ESF, cobertura vacinal, número de neoplasias intraepiteliais cervicais grau III diagnosticados). Resultados: Foram diagnosticados 2825 casos, a maioria nas macrorregiões de São Luís e Presidente Dutra, locais onde há maior média de ESF. Houve correlação estatisticamente significativa entre o número de diagnósticos desta neoplasia e IDH $(r=-0,646 ; p=0,048)$; número de equipes da ESF ( $r=-0,508 ; p=0,042)$; número de exames citopatológicos ( $r=0,567 ; p=0,005)$. Conclusão: Há tendência linear de decréscimo de casos nos últimos 9 anos. Da análise de variáveis socioculturais, sete macrorregiões não caracterizaram a escolaridade em mais de $70 \%$ dos exames. Há relação estatisticamente negativa e significativa, entre câncer do colo uterino e o IDH $(r=-0,646 ; p=0,048)$, e entre esta neoplasia e o número de $\mathrm{ESF}(r=-0,508 ; p=0,042)$, e sendo positiva e significativa, entre número de exames citopatológicos e número de neoplasias do colo uterino $(r=0,567 ; p=0,005)$.

Palavras-chave: Neoplasia intraepitelial cervical, Epidemiologia, Saúde da mulher.

\begin{abstract}
Objective: To identify and analyze the epidemiological profile of cervical cancer prior to the implementation of the HPV vaccination calendar in the State of Maranhão, from 2006 to 2014. Methods: Epidemiological data found in SISCOLO / DATASUS, and IBGE, were analyzed. eight health macro-regions, with the variables: sociodemographic indexes (education, race / color, HDI); health indicators (number of tests collected, number of FHS, vaccination coverage, number of grade III cervical intraepithelial neoplasms diagnosed). Results: 2825 cases were diagnosed, most of them in the São Luís and Presidente Dutra macroregions, places where there is a higher average of FHS. There was a statistically significant correlation between the number of diagnoses of this neoplasia and HDI $(r=-0.646 ; p=0.048)$; number of FHS teams $(r=-0.508 ; p=0.042)$; number of cytopathological exams $(r=0.567 ; p=0.005)$. Conclusion: There is a linear trend of decreasing cases in the last 9 years. From the analysis of socio-cultural variables, seven macro-regions did not characterize schooling in more than $70 \%$ of the exams. There is a statistically negative and significant relationship between cervical cancer and the HDI $(r=-0.646 ; p=0.048)$, and between this neoplasm and the number of FHS $(r=-0.508 ; p=0.042)$, and being positive and significant, between the number of cytopathological exams and the number of cervical neoplasms $(r=0.567 ; p=0.005)$.
\end{abstract}

Keywords: Cervical intraepithelial neoplasia, Epidemiology, Women's health.

1 Universidade Federal do Maranhão, Pinheiro - MA.

2 Universidade Federal do Maranhão, São Luís - MA. *E-mail: izolda.costa@hotmail.com

PUBLICADO EM: 2/2021 


\section{RESUMEN}

Objetivo: Identificar y analizar el perfil epidemiológico del cáncer cervicouterino previo a la implementación del calendario de vacunación contra el VPH en el estado de Maranhão, de 2006 a 2014. Metodos: Se analizaron los datos epidemiológicos encontrados en SISCOLO / DATASUS e IBGE. ocho macrorregiones de salud, con las variables: índices sociodemográficos (educación, raza / color, IDH); indicadores de salud (número de pruebas recogidas, número de ESF, cobertura de vacunación, número de neoplasias intraepiteliales cervicales grado III diagnosticadas). Resultados: Se diagnosticaron 2.825 casos, la mayoría de ellos en las macrorregiones São Luís y Presidente Dutra, lugares donde hay un mayor promedio de ESF. Hubo una correlación estadísticamente significativa entre el número de diagnósticos de esta neoplasia y el IDH ( $r=-0,646 ; p=0,048)$; número de equipos de la ESF $(r=-0,508 ; p=0,042)$; número de exámenes citopatológicos $(r=0,567 ; p=0,005)$. Conclusión: Existe una tendencia lineal de disminución de casos en los últimos 9 años. A partir del análisis de las variables socioculturales, siete macrorregiones no caracterizaron la escolaridad en más del $70 \%$ de los exámenes. Existe una relación estadísticamente negativa y significativa entre el cáncer de cuello uterino y el IDH $(r=-0,646 ; p=0,048)$, y entre esta neoplasia y el número de FHS $(r=-0,508 ; p=0,042)$, y ser positivo y significativo, entre el número de exámenes citopatológicos y el número de neoplasias cervicales $(r=0,567 ; p=0,005)$.

Palabras clave: Neoplasia intraepitelial cervical, Epidemiología, Salud de la mujer.

\section{INTRODUÇÃO}

O câncer do colo do útero ou cervical é o câncer pélvico mais comum entre as mulheres no Brasil (SADALLA JC, et al., 2015). Segundo o Instituto Nacional de Câncer José de Alencar Gomes da Silva (INCA), é o terceiro tumor mais frequente na população feminina e a quarta causa de morte de mulheres por câncer. Estima-se que cerca de $80 \%$ das mulheres sexualmente ativas irão adquirir o vírus ao longo de suas vidas (DALLA LIBERA LS, 2016). No Brasil, para o ano de 2019, foram esperados 16.370 novos casos de câncer do colo uterino, sendo 970 localizados no Estado do Maranhão (BRASIL, 2019a). A incidência desta neoplasia é consequência da exposição das mulheres a fatores de risco e da eficiência dos programas de rastreamento (BRASIL, 2013a).

Os fatores que contribuem para o desenvolvimento do câncer cervical são os relacionados aos aspectos socioeconômicos, estilo de vida e hábitos sexuais das mulheres. Uns dos exemplos que melhor pode confirmar esta afirmação é o que ocorre com os africanos que moram na África, e têm maior incidência de câncer do colo uterino do que africanos que vivem nos Estados Unidos, indicando que esta neoplasia está ligada diretamente ao estilo de vida socioeconômico (BRASIL, 2011).

Fatores como multiplicidade de parceiros, multiparidade, idade da primeira relação sexual, história reprodutiva da paciente (paridade, abortos, idade da menarca e da menopausa) e infecções ginecológicas repetidas, levam a um risco aumentado de desenvolver a neoplasia uterina (BRASIL, 2014). Quando se analisam esses eventos, observa-se que eles são considerados de risco, pois levam a contrair o principal senão o único fator de desencadeamento de câncer do colo uterino, o papiloma vírus humano. O Papiloma Vírus Humano (HPV) é uma doença sexualmente transmissível (DST), sendo que mulheres sexualmente ativas e que seguem esses pré-requisitos, têm o risco aumentado de contrair o vírus (BRASIL, 2014).

A infecção pelo HPV é considerada o principal fator de risco, bem como imunossupressão, iniciação sexual precoce, multiplicidade de parceiros, tabagismo, uso prolongado de contraceptivos orais, higiene íntima inadequada e baixas condições socioeconômicas (BRASIL, 2014; BRASIL, 2011). Aproximadamente 291 milhões de mulheres no mundo são portadoras do HPV, sendo que $32 \%$ estão infectadas pelos subtipos 16, 18 ou ambos (DALLA LIBERA LS, 2016). Comparando-se esse dado com a incidência anual de aproximadamente 500 mil casos de câncer do colo do útero, nota-se que o câncer é um desfecho raro, mesmo na presença da infecção pelo HPV. Ou seja, a infecção pelo HPV é um fator necessário, mas não suficiente, para o desenvolvimento do câncer cervical uterino (BRASIL, 2013a). 
Atualmente, a teoria mais aceita para a explicação do aparecimento do câncer do colo do útero correlaciona uma série de fatores considerados de risco com o papel central do papiloma vírus humano (HPV) (SILVA AK e ROSS JR, 2017). Desde 1992, a Organização Mundial de Saúde (OMS) considera que a persistência da infecção pelo Vírus HPV em altas cargas virais representa o principal fator de risco para o desenvolvimento da doença (WHO, 2008).

O principal meio de rastreamento desse tipo de câncer é realizado por meio do exame citológico do colo do útero, também conhecido como exame de Papanicolau. As alterações celulares são detectáveis e curáveis na maioria dos casos, o que justifica a importância da realização periódica deste exame entre a população feminina em idade reprodutiva, especialmente entre 25 e 59 anos (BRASIL, 2019a). O rastreamento deve ser feito a cada três anos, caso em dois anos seguidos seja apontado como normal, através do exame citopatológico (exame de Papanicolau), como recomenda a OMS (GONTIJO RC, et al, 2004; WHO, 2008).

O estadiamento do tumor é o principal fator prognóstico do câncer do colo do útero (CARMO CC e LUIZ RR, 2011). Nakagawa JT, et al. (2011) sugere que há uma relevante diferença na taxa de sobrevida nos países desenvolvidos, onde ocorre detecção precoce e melhor prognóstico, quando comparados aos países em desenvolvimento, onde a doença está sendo detectada somente na fase avançada.

Em vista disso, a informação sobre a cobertura, ou seja, o quantitativo da população de mulheres que realizaram os exames preventivos, e as taxas de acometimento pelo câncer do colo de útero são indispensáveis para a gestão dos serviços de saúde, sendo utilizadas nas atividades de planejamento da assistência à saúde da mulher, assim como na construção de indicadores de saúde e demográficos, tais como a taxa de mortalidade e cura (BRASIL, 2013a). E servem também para identificar necessidades específicas como, por exemplo, aumento da cobertura de exames preventivos e/ou aumento na cobertura vacinal (BRASIL, 2011).

Nesse sentido, os Sistemas de Informação em Saúde (SIS) são ferramentas importantes para conhecer a realidade, pois os dados produzidos por eles, além de permitirem análises epidemiológicas, subsidiam também decisões para a construção de política, planejamento, monitoramento e avaliação de programas de saúde (JESUS A, et al., 2018).

Em 1988 foram desenvolvidas as primeiras ferramentas gerenciais adequadas para a informação e acompanhamento de programas de controle do câncer do colo do útero, no Brasil, denominado Sistema de Informação do Controle do Câncer do Colo do Útero (SISCOLO), reunindo informações sobre a população feminina, bem como resultados de exames, seguimento de casos alterados e outras inerentes ao tema. Estas ferramentas foram desenvolvidas pelo INCA, em parceira com o Departamento de Informática do Sistema Único de Saúde (DATASUS), sendo que, em 2008, estas instituições também desenvolveram o Sistema de Informação do Controle do Câncer de Mama (SISMAMA) (BRASIL, 2011). Ambos sistemas trazem informações onde é possível conhecer o perfil epidemiológico das mulheres que realizaram o exame preventivo, bem como as condições referentes à assistência em saúde oferecida a esse grupo (ANDRADE SM, et al., 2017).

Em 2006 foi aprovada pela Food and Administration (FDA) a vacina contra o HPV referente aos sorotipos 6, 11, 16 e 18 (quadrivalente). Dois anos depois a vacina contra apenas os sorotipos 16 e 18 (bivalente) foi autorizada (BRASIL, 2016). Até 2013 os serviços fornecedores eram apenas os privados. Em 2014, o Ministério da Saúde, através da Portaria $n^{\circ} 54$, de 18 de novembro de 2013, introduziu a vacina quadrivalente no calendário vacinal em 2014, com o objetivo de prevenir o desenvolvimento da neoplasia cervical, oferecendo distribuição gratuita no Sistema Único de Saúde (SUS) (BRASIL, 2013b). Atualmente, ela é ofertada em todas as unidades de saúde pública no território brasileiro para ambos os sexos e, em conjunto com a adoção de estratégias de imunização também nas escolas públicas e particulares, há ainda a educação em saúde, sujeita a intervenção direta do planejamento adotado pelas esferas envolvidas (poder Municipal, Estadual e União) (SILVA AK e ROSS JR, 2017; BRASIL, 2016).

Diante do exposto, este estudo tem como objetivo identificar e analisar o perfil epidemiológico do câncer do colo do útero anterior à implantação do calendário vacinal para o HPV no Estado do Maranhão, por macrorregiões de saúde, no período de 2006 a 2014. 


\section{MÉTODOS}

Trata-se de um estudo descritivo, retrospectivo, de abordagem quantitativa, com o objetivo de descrever informações epidemiológicas sobre a realização do exame Papanicolau e o desenvolvimento do câncer do colo do útero, anterior à implantação da cobertura vacinal para o HPV, no período de 2006 a 2014.

Realizou-se consulta direta aos domínios públicos do Instituto Brasileiro de Geografia e Estatísticas (IBGE), dados informatizados do Programa Nacional de Imunização (PNI) e do SISCOLO, plataforma desenvolvida pelo departamento de informática do SUS, em parceria com o INCA, e voltado para a estruturação do programa Viva Mulher, que coleta e processa informação sobre identificação de pacientes e laudos de exames cito e histopatológicos (IBGE, 2017; BRASIL, s/d).

Os dados coletados são referentes às oito macrorregiões de saúde do Estado do Maranhão, proposta pela Resolução CIB/MA № 44/2011 de junho de 2011, sendo elas compostas por oito macrorregiões, que representam os principais polos de saúde do Estado e desempenham papel essencial na organização das redes de atenção à saúde (MARANHÃO, 2012).

As informações coletadas nos bancos de dados anteriormente citados, foram divididas em duas categorias: 1) índices sociodemográficos (escolaridade, raça/cor e IDH); 2) indicadores em saúde (número de exames coletados, número de equipes saúde da família, cobertura vacinal e número de neoplasias intraepiteliais cervicais grau III diagnosticados).

Utilizou-se o ano de coleta de dados a partir de 2006 por compreender ao início da disponibilização dos dados pelo SISCOLO e, em outro extremo, o ano de 2014, data que coincide com a implantação do calendário vacinal para o HPV na rede pública efetivado no Brasil, sendo que tal proposta de busca de dados pode servir de base para comparações futuras, principalmente para identificar se houve alteração da incidência a partir das campanhas de imunização.

A organização e tabulação dos dados foi realizada utilizando o programa Microsoft Office Excel 2016 a partir da importação através do SISCOLO/DATASUS, e a análise estatística se deu por meio do programa IBM SPSS Statistics 20.

Foi utilizado o teste estatístico Shapiro-Wilk para identificar o padrão de Normalidade da amostra. Para as variáveis que tiveram distribuição normal utilizou-se o teste de correlação de Pearson. Adotou-se nível de significância de 95\% para a rejeição da hipótese nula $(p<0,05)$ (FIGUEIREDO FILHO DB e SILVA JÚNIOR JA, 2009).

O grau de associação entre as variáveis analisadas (número de diagnósticos de câncer do colo uterino e o número de equipes saúde da família, por exemplo) entre outras relações teve como base a classificação de Dancey C e Reidy J (2006), ou seja, tem-se que o coeficiente de Pearson ( $r$ ) varia no intervalo de [-1 a 1] e tomar-se-á então que, positivo ou negativo: $r=0,10$ até 0,30 (fraco); $r=0,40$ até 0,6 (moderado); $r=0,70$ até 1 (forte).

No desenvolvimento desta pesquisa foram tomados os devidos cuidados éticos presentes nas Resoluções 466/12 e 580/2018, do Conselho Nacional de Saúde, do Ministério da Saúde, sinalizando a dispensa por apreciação do Comitê de Ética em Pesquisa, por se tratar de utilização de dados públicos presentes no DATASUS (BRASIL, 2013c).

\section{RESULTADOS}

De acordo com os dados obtidos através de consulta ao SISCOLO (2019), observou-se que no período entre 2006 a 2014 foram diagnosticados 2.825 casos de câncer do colo do útero nas oito macrorregiões de saúde do Estado do Maranhão. Dos anos em questão, os anos de 2010 e 2012 apresentaram, respectivamente, maior e o menor número de neoplasias diagnosticadas, correspondendo a 555 e 187 casos. Observa-se também tendência linear de decrescimento do número de casos de neoplasia intraepitelial cervical nos últimos 9 anos (Gráfico 1). 
Gráfico 1 - Número de diagnósticos de neoplasia do colo do útero e previsão linear no Estado do Maranhão, no período de 2006 a 2014.

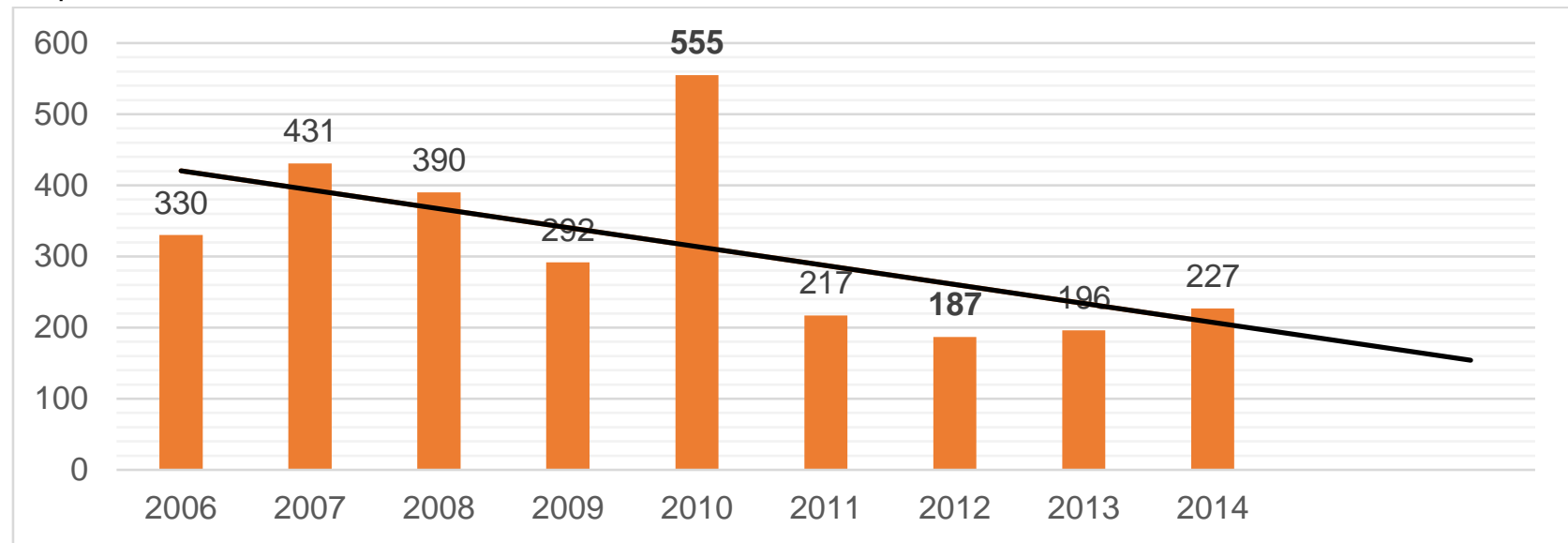

Fonte: Nogueira LM, et al., 2020. Baseado em: SISCOLO/DATASUS/MS, BRASIL - 2006 a 2014.

Quanto à caracterização das mulheres que realizaram o exame citopatológicos, as macrorregiões de São Luís e Presidente Dutra apresentaram a maior quantidade de diagnósticos com média anual de 66,22 e 58,33 de casos de NIC III, respectivamente. Em contrapartida, a macrorregião de Balsas apresentou a menor média anual com 6,33, ou seja, em um recorte de 9 anos, houve 57 casos diagnosticados com NIC grau III nessa macrorregião (Tabela 1).

Outro importante parâmetro analisado através dos sistemas de informação do Ministério da Saúde, diz respeito à média de exames citopatológicos realizados nesse período, sendo que dentre as macrorregiões de saúde, destacam-se Coroatá $(111.500,5)$, São Luís $(93.600,6)$ e Presidente Dutra $(51.388,1)$.

Da análise de variáveis socioculturais, verificou-se ainda, através da frequência média, que as macrorregiões de São Luís, Imperatriz, Pinheiro, Balsas, Caxias, Presidente Dutra e Santa Inês deixaram de caracterizar, no momento de realização do exame citopatológico, a escolaridade em mais de $70 \%$ das vezes que as pacientes foram realizar o exame. A macrorregião de Coroatá teve taxa de informações ignoradas inferiores às demais, embora deixando ainda de caracterizar as pacientes em cerca de $60,6 \%$ dos casos. Excetuando-se esse achado, observou-se que a maioria tinha o ensino fundamental incompleto.

No Cadastro Nacional dos Estabelecimentos de Saúde do Brasil (CNES) é possível verificar o número de equipes da ESF por região de saúde, em cada macrorregião. Observa-se que a média de equipes ESF, no período de 2006 a 2014 é maior nas macrorregiões de São Luís e Presidente Dutra $(530,8$ e 259,1) que são as macrorregiões com o maior contingente populacional (IBGE, 2017). Verifica-se também menor média do número de equipes ESF nas macrorregiões de Caxias e Balsas $(207,1$ e 81,6) (BRASIL, 2019d).

Observa-se também que na macrorregião de São Luís, a relação entre o número de equipes por habitantes é de aproximadamente uma equipe para cada 4.377 pessoas. A macrorregião de Imperatriz apresenta relação de 1 equipe para cada 2.456 habitantes.

A média do índice de desenvolvimento humano das macrorregiões no período de 2006 a 2014 mostrou discrepâncias. Nesse sentido, observou-se que a macrorregião de São Luís apresentou maior IDH (0,691), por outro lado, a macrorregião de Coroatá, composta por trinta municípios, entre eles Codó, Bacabal, Trizidela do Vale e Pedreiras, apresentou menor média de IDH $(0,547)$.

Quanto à distribuição de casos diagnosticados com neoplasia intraepitelial grau III nas oito sedes das macrorregiões de saúde, a macrorregião de Presidente Dutra teve o maior pico de casos confirmados da doença no ano de 2010 (260 casos). A respeito do número absoluto de casos confirmados nos últimos nove anos, destaca-se a macrorregião de São Luís com 596 casos diagnosticados, seguidos por Presidente Dutra com 525 e a macrorregião de Pinheiro com 447. As macrorregiões que apresentaram menor quantidade de casos, entre os anos de 2006 a 2014, foram Balsas e Caxias, com 57 e 248 diagnósticos respectivamente (Gráfico 2). 
Gráfico 2 - Distribuição de casos diagnosticados com NIC III nas oito Macrorregiões de Saúde do Estado do Maranhão no período de 2006 a 2014.

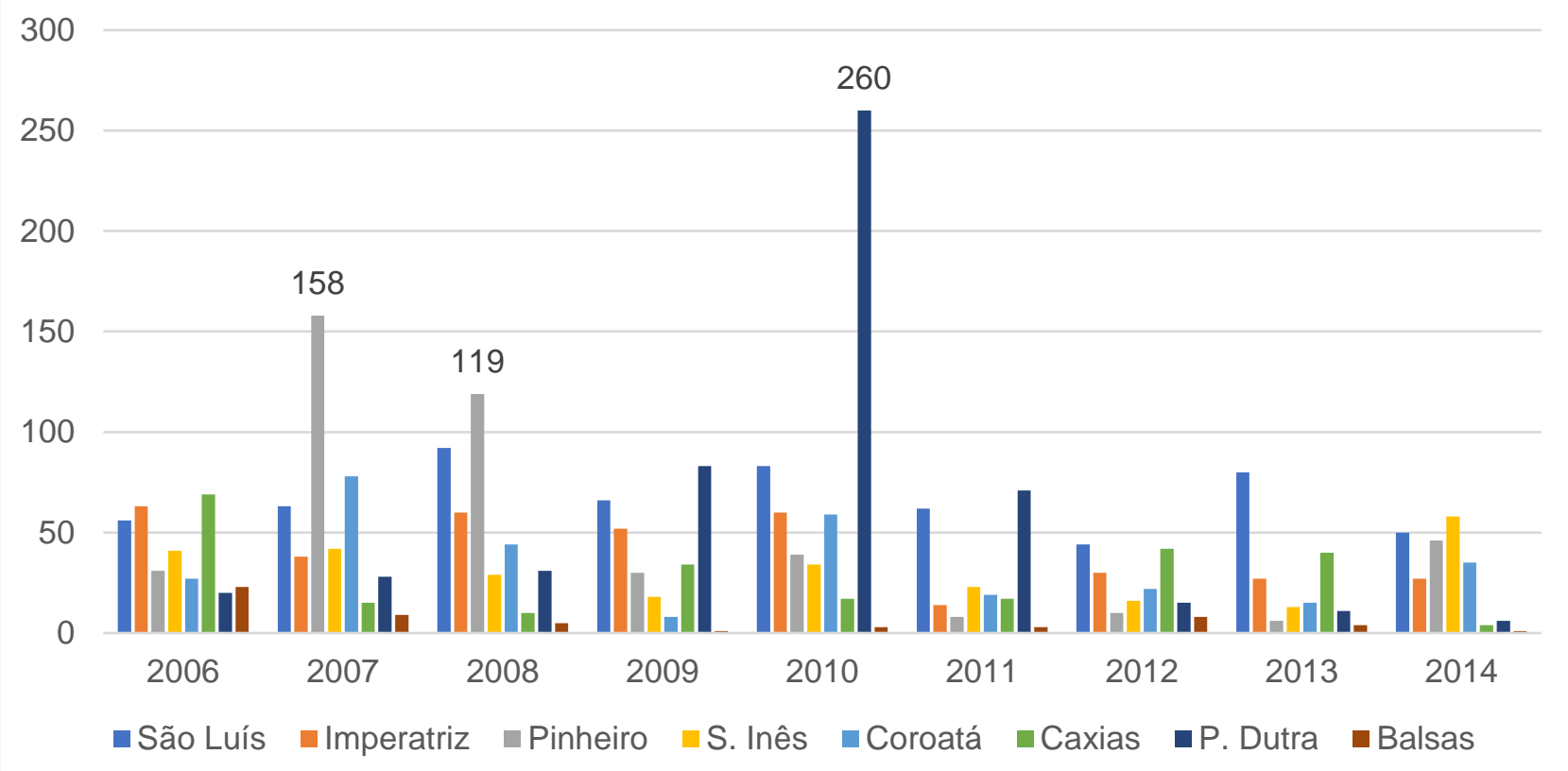

Fonte: Nogueira LM, et al., 2020. Baseado em: SISCOLO/DATASUS/MS, BRASIL - 2006 a 2014.

As macrorregiões de São Luís e de Presidente Dutra, além de possuírem a maior média de equipes da ESF, apresentam também uma maior média do número de diagnóstico de NIC III (Gráfico 3).

Gráfico 3 - Distribuição da média de número de equipes de saúde e média de casos diagnosticados de NIC III, nas oito macrorregiões de saúde do Maranhão, no período de 2006 a 2014.

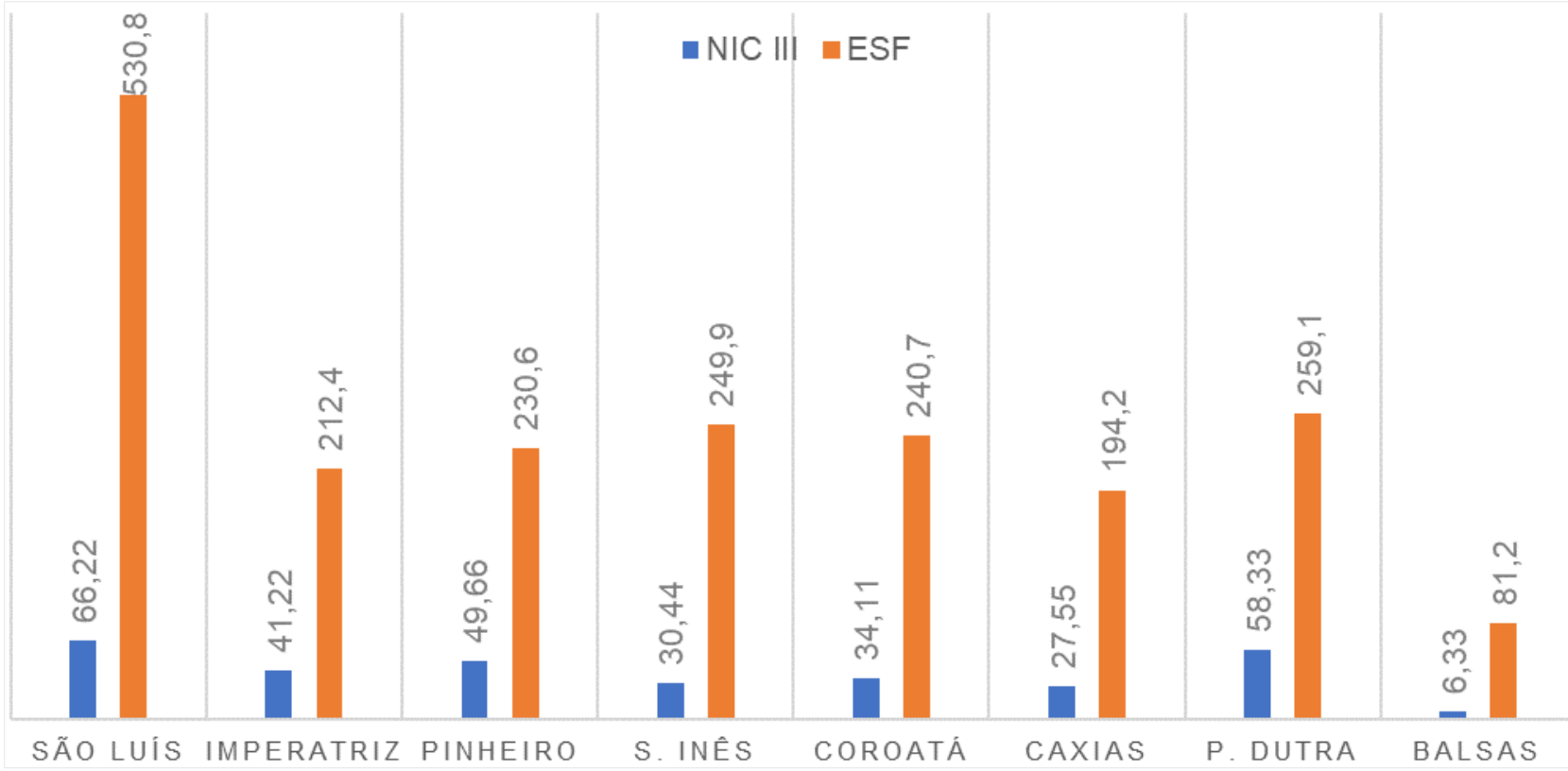

Fonte: Nogueira LM, et al., 2020. Baseado em: SISCOLO/DATASUS/MS, BRASIL - 2006 a 2014.

Ao analisar-se a correlação entre o número de diagnósticos de neoplasia intraepitelial cervical grau III e as variáveis sociodemográficas (escolaridade e IDH) e da saúde (número de ESF e número de exames citopatológicos) no Estado do Maranhão, observou-se através do teste de correlação de Pearson, uma relação estatisticamente negativa e significativa, de moderada intensidade, entre câncer do colo uterino e o IDH ( $r=-$ 0,$646 ; p=0,048$ ). $O$ teste não demonstrou correlação entre a neoplasia e a escolaridade (Tabela 2). 
No que tange às variáveis relacionadas à saúde, observou-se correlação estatisticamente negativa e significativa, de moderada intensidade, entre a neoplasia e o número de equipes saúde da família ( $r=-0,508$; $p=0,042)$. Com relação ao número de exames citopatológicos realizados, houve correlação estatisticamente positiva e significativa, de moderada intensidade, com o número de neoplasias do colo uterino ( $r=0,567$; $\mathrm{p}=0,005)$.

Tabela 2 - Correlação das variáveis sociodemográficas e da saúde com o número de casos diagnosticados com Neoplasia Intraepitelial grau III, no Estado do Maranhão (2006-2014).

\begin{tabular}{lcc}
\hline & \multicolumn{2}{c}{ NIC III } \\
\cline { 2 - 3 } & r-Valor & $\mathbf{p}$ \\
\hline IDH & $-\mathbf{0 , 6 4 6}$ & $\mathbf{0 , 0 4 8}$ \\
\hline Escolaridade (anos) & & \\
$<8$ & 0,523 & 0,315 \\
$\geq 8$ e $\leq 11$ & 0,125 & 0,450 \\
$\geq 12$ & 0,256 & 0,653 \\
\hline Número de ESF & $-\mathbf{0 , 5 0 8}$ & $\mathbf{0 , 0 4 2}$ \\
\hline Número de Exames citopatológicos & $\mathbf{0 , 5 6 7}$ & $\mathbf{0 , 0 0 5}$ \\
\hline
\end{tabular}

Fonte: Nogueira LM, et al., 2020. Baseado em: SISCOLO/DATASUS/MS, BRASIL - 2006 a 2014.

\section{DISCUSSÃO}

Nesta pesquisa, foram encontrados 2.825 casos de câncer do colo do útero, sendo que dados desta natureza, entre outros objetivos, tem como propósito auxiliar na formulação de políticas públicas de saúde, no planejamento das ações de prevenção e nos estudos epidemiológicos que buscam traçar o perfil da assistência prestada às pacientes diagnosticadas com câncer (THULER LC, et al., 2012).

Este estudo demonstrou tendência de decrescimento dos casos diagnosticados com NIC III, corroborando com Muniz GG, et al. (2019) que afirmam que a diminuição da incidência de câncer do colo uterino é proporcional à prevenção por meio do exame de Papanicolau, desde que existam programas efetivos e organizados. Também vai ao encontro de Viscondi JYK (2017), que relata que o decréscimo do número de diagnósticos de neoplasia intraepitelial pode ocorrer com o aumento do acompanhamento das mulheres pelos sistemas de saúde e a efetividade do seguimento para lesões pré-malignas. Isto pode justificar o aumento do número de diagnósticos no ano de 2007 e 2008 na macrorregião de Pinheiro e em 2010 na de Presidente Dutra, que pode estar relacionado ao aumento da procura de exames nesse período.

Ademais, os resultados desta pesquisa indicam que maioria das mulheres que realizou os exames citopatológicos apresentou baixo nível de escolaridade, excluindo os dados ignorados por falta de informação na coleta do exame, havendo predomínio do ensino fundamental incompleto como nível de escolaridade mais prevalente na maioria das macrorregiões, indo ao encontro dos achados de Silva DSM, et al. (2014), e Melo SCCS, et al. (2009).

No presente estudo, observou-se correlação negativa entre o número de casos diagnosticados com neoplasia uterina e o IDH em todas as macrorregiões analisadas, ou seja, uma relação inversamente proporcional, pois na medida em que o índice aumenta, diminui o número de casos e vice-versa. Tal associação, assim como Soneji S e Fukui N (2013), e Lopes VAS e Ribeiro JM (2019) destacam, está atrelada aos fatores culturais, socioeconômicos e de assistência ao serviço de saúde, principalmente público. Sadovsky ADI, et al. (2015) ressaltam ainda que o IDH mais baixo está associado ao aumento da mortalidade por câncer do colo do útero.

Este estudo indica que há correlação negativa, estatisticamente significativa, entre a neoplasia por câncer do colo do útero e o número de equipes saúde da família em todas as macrorregiões estudadas, ou seja, na 
medida em que aumentam o número de equipes, há tendência de diminuição do agravo. Nesse sentido, o modelo de assistência empregado através da ESF, favorece o acesso da população ao programa de rastreamento do câncer do colo do útero (GIRIANELLI VR, et al., 2014). No Brasil, as equipes da ESF cumprem um papel importante no rastreio dessa doença (FARNESE JM e HOFFMANN EJ, 2013).

A estabilidade da oferta do exame citopatológico (Papanicolau) é um ponto chave das ações de rastreamento, de modo que a cobertura da população alvo em torno de $80 \%$ a $100 \%$ por esse exame, associada a uma rede organizada para diagnóstico e tratamento adequado, pode reduzir em média $60 \%$ a $90 \%$ as formas invasivas de câncer na população, a exemplo das neoplasias intraepiteliais cervicais de grau três (GIRIANELLI VR, et al., 2014). Neste estudo observou-se que o aumento do número de exames realizados esteve correlacionado com o aumento do número de neoplasias do colo uterino nas macrorregiões verificadas.

Sendo a região Nordeste (sobretudo o Estado do Maranhão, alvo desta nossa pesquisa) uma das que apresenta as menores taxas de PIB no país segundo o IBGE (2017), torna-se necessário analisar mais profundamente esta variável sociodemográfica, afim de traçar mecanismos cada vez mais efetivos para a diminuição da incidência deste agravo, visto que a partir de 2020, estima-se que ocorrerão anualmente cerca de 15 milhões de novos casos de câncer no mundo, dos quais cerca de 70\% ocorrerão em países que não dispõem de recursos para seu controle e rastreamento (WHO, 2008).

Por este motivo, ressalta-se a importância dos métodos de rastreamento e prevenção do câncer do colo do útero, tal como a amplificação das campanhas de vacinação contra o HPV e maior conscientização em educação em saúde sobre as questões relacionada aos fatores de risco nessa região.

Nesta pesquisa, entretanto, há limitação de estudo, por se tratar de dados secundários obtidos por meio da digitação dos resultados dos exames preventivos no site do SISCOLO no DATASUS (BRASIL, s/d), com possibilidade de subnotificação, seja por limitações técnicas do exame ao não diagnosticar a doença, seja por dados não registrados por limitações sobre a não realização do preventivo.

\section{CONCLUSÃO}

As variáveis socioeconômicas e de saúde estão relacionadas com o câncer do colo do útero no Estado do Maranhão. No período analisado, foram diagnosticados 2.825 casos nas oito macrorregiões, com maior número de diagnósticos onde há maior média de equipes da ESF, com tendência linear de decrescimento do número de casos nos últimos 9 anos. Também há relação estatisticamente negativa e significativa entre câncer do colo uterino e o IDH ( $r=-0,646 ; p=0,048)$, e entre a neoplasia e o número de $E S F(r=-0,508$; $\mathrm{p}=0,042$ ). Assim, é recomendado que os métodos para prevenção desse desfecho se intensifiquem a fim de promover melhores indicadores de saúde da mulher, na região.

\section{REFERÊNCIAS}

1. ANDRADE SM, et al. Epidemiologia e Indicadores de Saúde. In: ANDRADE SM, et al (Org.), Bases da saúde coletiva, 2. ed. Paraná: Eduel. 2017; 10: 183-210.

2. BRASIL. Ministério da Saúde. INCA - Instituto Nacional de Câncer José Alencar Gomes da Silva. 2019. Disponível em: http://www.inca.gov.br/wps/wcm/connect/tiposdecancer/site/home/colo_utero. Acesso em: 15 nov 2019.

3. BRASIL. Ministério da Saúde. Instituto Nacional de Câncer. Sistema de informação do controle do câncer de mama (SISMAMA) e do câncer do colo do útero. Manual Gerencial. Rio de Janeiro: INCA, 2011; 116.

4. BRASIL. Instituto Nacional de Câncer. HPV e câncer Perguntas mais frequentes. 2016. Disponível em: http://www1.inca.gov.br/conteudo_view.asp?id=2687. Acesso em: 15 set. 2020.

5. BRASIL. MINISTÉRIO DA SAÚDE. Guia prático sobre HPV: Guia de Perguntas e Respostas para Profissional de Saúde. Brasília. 2014; 14 p.

6. BRASIL. Ministério da Saúde. DATASUS/SISCOLO - Departamento de Informática do SUS. Disponível em: http://www2.datasus.gov.br/DATASUS/index.php?area=060303. Acesso em: 15 nov 2019.

7. BRASIL. Ministério da Saúde. SI-PNI Programa Nacional de Imunização. Disponível em: http://pni.datasus.gov.br/consulta_hpv_14_selecao.php. Acesso em 15 set 2020.

8. BRASIL_a. Ministério da Saúde. Instituto Nacional do Câncer. Sistema de informação do câncer. Manual preliminar de apoia à implantação. Rio de Janeiro: INCA, 2013.

9. BRASIL b. Ministério da Saúde. Portaria n 54 de 18 de janeiro de 2013. Torna pública a decisão incorporar a vacina quadrivalente contra HPV na prevenção do câncer de colo do útero no Sistema Único de Saúde-SUS, 2013. 
10. BRASIL_c. Ministério da Saúde. Conselho Nacional de Saúde. Resolução no 466 de 12 de dezembro de 2012. Dispõe sobre as diretrizes e normas regulamentadoras de pesquisas envolvendo seres humanos. Diário Oficial da União da República Federativa do Brasil, junho de 2013.

11. BRASIL d. Ministério da Saúde. Secretaria de Atenção à Saúde. Cadastro Nacional dos Estabelecimentos de Saúde do Brasil - CNES. 2019. Disponível em: http://cnes2.datasus.gov.br/. Acesso em 15 set 2020.

12. CARMO CC, LUIZ RR. Survival of a cohort of women with cervical cancer diagnosed in a Brazilian cancer center. Revista de Saúde Pública, 2011; 45(4): 661-667.

13. DALLA LIBERA LS. Avaliação da infecção pelo Papiloma Vírus Humano (HPV) em exames citopatológicos. Brazilian Journal of Clinical Analysis, 2016; 48(2): 138-143.

14. DANCEY C, REIDY J. Estatística sem matemática para psicologia: Usando SPSS para Windows. Porto Alegre, Artmed; 2006; 608.

15. MELO SCCS, et al. Alterações citopatológicas e fatores de risco para ocorrência do câncer de colo uterino. Revista Gaúcha de Enfermagem, 2009; 30(4): 602.

16. FARNESE JM, HOFFMANN EJ. Avaliação do Rastreamento Para Câncer de Colo Uterino em Duas Equipes de Estratégia Saúde da Família em Montes Claros, Minas Gerais, Brasil. Revista Baiana de Saúde Pública, 2013; 37(1): 34.

17. FIGUEIREDO FILHO DB, SILVA JÚNIOR JA. Desvendando os Mistérios do Coeficiente de Correlação de Pearson (r). Revista Política Hoje, 2009; 18(1): 115-146.

18. GIRIANELLI VR, et al. Adesão ao rastreamento para câncer do colo do útero entre mulheres de comunidades assistidas pela Estratégia Saúde da Família da Baixada Fluminense, Rio de Janeiro, Brasil. Revista Brasileira de Ginecologia e Obstetrícia, 2014; 36(5): 198-204.

19. GONTIJO RC, et al. Evaluation of alternative methods in cervical screening: HPV DNA detection and visual inspection. Revista Brasileira de Ginecologia e Obstetrícia, 2004; 26(4): 269-275.

20. INSTITUTO BRASILEIRO DE GEOGRAFIA E ESTATÍSTICA - IBGE: Cidades. 2017. Disponível em: <https://cidades.ibge.gov.br/brasil/se/aracaju/panorama> Acesso em 15 set 2020.

21. JESUS A, et al. Investigação de informação e comunicação: o boletim epidemiológico como dispositivo interdisciplinar de saúde. Dissertação (Mestrado em Informação e comunicação em saúde). Fundação Osvaldo Cruz. Rio de Janeiro. $2018 ; 111$.

22. LOPES VAS, RIBEIRO JM. Fatores limitadores e facilitadores para o controle do câncer de colo de útero: uma revisão de literatura. Ciência \& Saúde Coletiva, 2019; 24: 3431-3442.

23. MARANHÃO. RESOLUÇÃO DA COMISSÃO INTERGESTORES BIPARTITE (CIB/MA) № 44/2011 de 16 de junho de 2012. Dispõe sobre a conformação de 19 Regiões de Saúde e de 08 Macrorregiões.

24. MUNIZ GG, et al. Prosseguimento de estudo de coorte histórica de pacientes com biópsias de colo uterino alteradas e prognóstico no Distrito Federal de 2012 a 2018. Programa de Iniciação Científica-PIC/UniCEUB-Relatórios de Pesquisa, 2019; 4(1): 3-47.

25. NAKAGAWA JT, et al. Carcinoma do colo do útero: taxa de sobrevida e fatores prognósticos em mulheres no Estado de Mato Grosso. Acta Paulista de Enfermagem, 2011; 24(5): 631-637.

26. SADALLA JC, et al. Cervical cancer: what's new?. Revista da Associação Médica Brasileira, 2015; 61(6): 536-542.

27. SADOVSKY ADI, et al. Human Development Index and secondary prevention of breast and cervical cancer: an ecological study. Cadernos de Saúde Pública, 2015; 31(7): 1539-1550.

28. SILVA AK, ROSS JR. Vacinação contra o Papiloma Vírus Humano no Brasil: uma interlocução com as publicações científicas. JMPHC- Journal of Management \& Primary Health Care, 2017, 8(1): 91-98.

29. SILVA DSM, et al. Rastreamento do câncer do colo do útero no Estado do Maranhão, Brasil. Ciência \& Saúde Coletiva, 2014; 19: 1163-1170.

30. SONEJI S, FUKUI N. Socioeconomic determinants of cervical cancer screening in Latin America. Revista Panamericana de Salud Pública, 2013; 33: 174-182.

31. THULER LCS, et al. Perfil das pacientes com câncer do colo do útero no Brasil, 2000-2009: estudo de base secundária. Revista Brasileira de Cancerologia, 2012; 58 (3): 351-357.

32. VISCONDI JYK. Análise de custo-efetividade de estratégias de rastreamento do câncer do colo do útero no Brasil. Tese (Doutorado em Ciências) Faculdade de Medicina da Universidade de São Paulo. 2017; 161.

33. WORLD HEALTH ORGANIZATION. WHO programme on cancer control. Developing a global strategy for cancer. Lyon: WHO; 2008; 3. 